\title{
Simulation of serrated yielding with noise effects included
}

\author{
K NEELAKANTAN and G VENKATARAMAN \\ ANURAG, Advanced Numerical Research and Analysis Group, Kanchanbagh, \\ Hyderabad 500258 , India \\ MS received 31 May 1991
}

\begin{abstract}
The phenomenon of repeated yield drops is extensively studied using computer simulation. The basic input is the Cottrell-Bilby model for dislocation drag, used in conjunction with a suitable scenario for work hardening. Stochastic effects associated with dislocation velocity and density are built in via multiplicative noise. Using the above scheme for modelling the plastic response and assuming first that the yielding is homogeneous, the machine equation is solved for successive time steps to obtain complete stress-strain curves, similar to those obtained in laboratory experiments. Real-life patterns are well simulated, including several detailed features observed in diverse experiments. The role of noise in the region of marginal stability is explored. The simulation study is next extended to the case of inhomogeneous yielding where, in addition to the model earlier proposed by Penning. we explore one which takes some account of dislocation flow across the sample. Attention is then drawn to similarities observed in the flow of sand in a sand pile. Finally, some suggestions are made for further work.
\end{abstract}

Keywords. Serrated yielding; inhomogeneous yielding; jerky flow; simulation.

\section{Introduction}

The phenomenon of repeated yielding during tensile-test experiments, also known variously as jerky flow, serrated yielding etc. has been extensively studied (e.g. Keh et al 1968; Brindley and Worthington 1970; Hall 1970; Baird 1973; Kocks 1981; Rodriguez 1984; Kocks et al 1985).

Depending upon the material used and its previous history on the one hand, and the conditions of the experiment on the other, different physical mechanisms might be responsible for the jerky flow (Rodriguez 1984). Nevertheless, a common underlying point is that repeated yielding arises basically due to a "negativeresistance" feature in the flow stress characteristics.

In an earlier paper (Neelakantan and Venkataraman 1983) we employed analog electronic simulation to comprehensively delineate the principal features arising out of the above mentioned negative resistance, features which are also observed in experiments. We report here the results of further simulation studies, this time performed using a digital computer. While such a computer simulation study is in itself not new (for example, Kubin et al (1982) have carried out a simulation study earlier), a significant feature of our work is the incorporation of underlying stochastic processes intrinsic to yielding. As a result, the yield patterns simulated by us closely resemble those obtained in many experiments, especially in relation to irregularities in yielding. In contrast to studies in physics, the role and the relevance of stochastic processes in metallurgical phenomena have hitherto been generally ignored, with the possible exception of anelastic relaxation (-for an introductory account on the role fluctuations in mechanical relaxation, see Venkataraman 1979 
and 1982). Our work strongly suggests that laboratory experiments on the noise aspects of yielding would be most rewarding.

It is widely recognized that serrated yielding is a process which is not homogeneous across the sample. However, barring qualitative and semiquantitative remarks concerning the role of inhomogeneity, no serious theoretical study of it appears to have been carried out so far. This is not surprising since the concerned equations are rather complex, added to which perhaps is the fact that reliable models for inhomogeneous yielding are not available. Our work includes a modest effort in simulation studies in this largely uncharted area; hopefully it would spur further work by others, especially in modelling.

The plan of the paper is as follows: We begin by first calling attention in $\S 2$ to the role played by the negative-resistance feature alluded to earlier. We then describe in $\S 3$ our simulation scheme, following which we present various results. To start with, we report in $\S 4$ results obtained by assuming (purely for convenience) that the yielding is homogeneous throughout the sample. These give a feel for how a wide variety of observed patterns could be easily and conveniently simulated. More important, the homogeneity assumption permits a ready and convenient analysis of the statistics of spikes observed during the yielding process.

As already remarked, the assumption that yielding is homogeneous is rather drastic and accordingly we relax this assumption in $\S 5$. Besides presenting (for the first time we believe) various simulation results pertaining to inhomogeneous yielding, we also try to relate our results to recent studies (performed via cellular automata) on phenomena-like earthquakes and avalanches. Possibilities for future work and some concluding remarks are made in $\S 6$, wrapping up the paper.

\section{The role of the negative-resistance feature}

The current-voltage characteristics of many electronic devices have a nonmonotonic behaviour, and it is well-known that such devices can be used to generate electrical oscillations. In a perceptive study, Franck (1974) has called attention to the fact that a wide variety of oscillatory phenomena could be traced to the existence of such nonmonotonic characteristic curves or curves with what we refer to as a negative-resistance feature (for a convenient paraphrase of Franck's work especially in relation to mechanical behaviour, see Venkataraman 1982).

In the context of serrated yielding, the relevant characteristic curve is that which relates flow stress $\sigma$ to (plastic) strain rate $\dot{\varepsilon}_{p}$ or, equivalently, the flow stressdislocation velocity $(\sigma-v)$ curve. Starting with the work of Penning (1972), there has been, a growing appreciation of the role of the so-called negative-resistance feature, and since it plays such a crucial role in producing serrations it is pertinent to offer a brief comment on it. One important feature of the $\sigma-\dot{\varepsilon}_{p}$ curve is that unlike in electronics, it depends on the state of strain of the material. Thus the characteristic curve may be said to evolve during the course of the experiment, and the interpretation of observed phenomena must be done against the background of such an evolutionary process.

A qualitative feel for all this may be had from figures 1 and 2 . In figure 1 is illustrated the usual type of $\sigma$ versus $\dot{\varepsilon}$ curve. The load line is determined by the applied strain rate which, using the jargon of electronics, we sometimes refer to as the applied bias. It is well-known there are oscillations when the load line intersects 


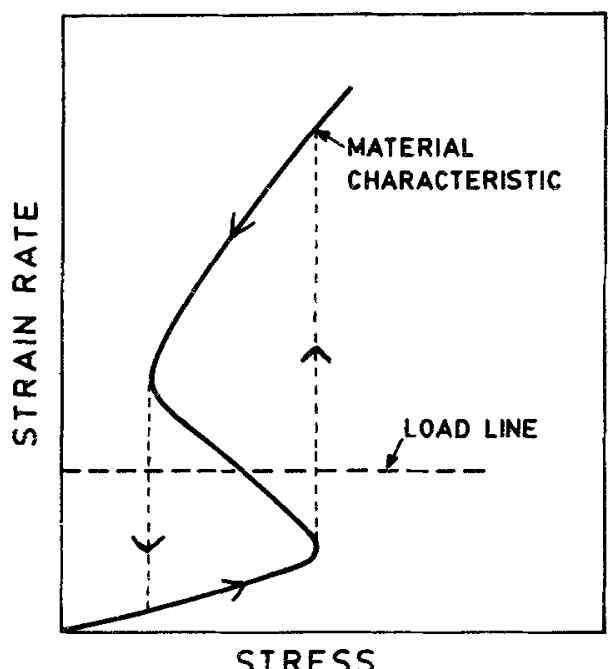

Figure 1. Schematic representation of the stress $(\sigma)$ versus strain rate $(\dot{\varepsilon})$ behaviour. Also shown is the load line whose position depends on the applied strain rate. If, as indicated here, the load line intersects in the negative-resistance region, the state of the system follows the path of the arrows, leading to oscillations. The modification to this picture due to the evolution of the material characteristic is discussed in the next figure.

the characteristic curve as indicated, with the state of the system moving along the cyclic path shown by the arrows. In our situation we have to deal with a family of curves since the material characteristic depends on the state of strain. This then essentially results in a surface as shown in figure 2 . As the tensile testing progresses, the state of the system criss crosses over this undulating terrain in turn leading to serrations, provided the bias is in the unstable region. Clearly, the serrations last only as long as the intersection of the load line with the characteristic curve is in the negative-resistance region. As a result, serrations occur only for certain range of parameters (see, for example, Mulford and Kocks 1979; Neelakantan and Venkataraman 1983; Strudel 1984).

\section{The model and the simulation scheme}

In this section, we outline the model used in our studies as also the simulation scheme employed. The present discussion is restricted to homogeneous yielding; inhomogeneous yielding will be considered in a later section.

The mathematical analysis of the outcome of a tensile-test experiment requires a combination of the so-called machine equation with one or more equations which describe the thermomechanical state of the material being studied. The machine equation is given by

$$
\dot{\varepsilon}_{a}=\left(\dot{\sigma} / K_{\text {eff }}\right)+\dot{\varepsilon}_{p},
$$

where $\dot{\varepsilon}_{a}$ is the applied strain rate, $\sigma$ the stress developed, $K_{\text {eff }}$ an effective stiffness and $\varepsilon_{p}$ the plastic strain. The above equation must be supplemented by a suitable one for $\dot{\sigma}$ and one for $\dot{T}$ the time rate of change of the temperature $T$ of the 

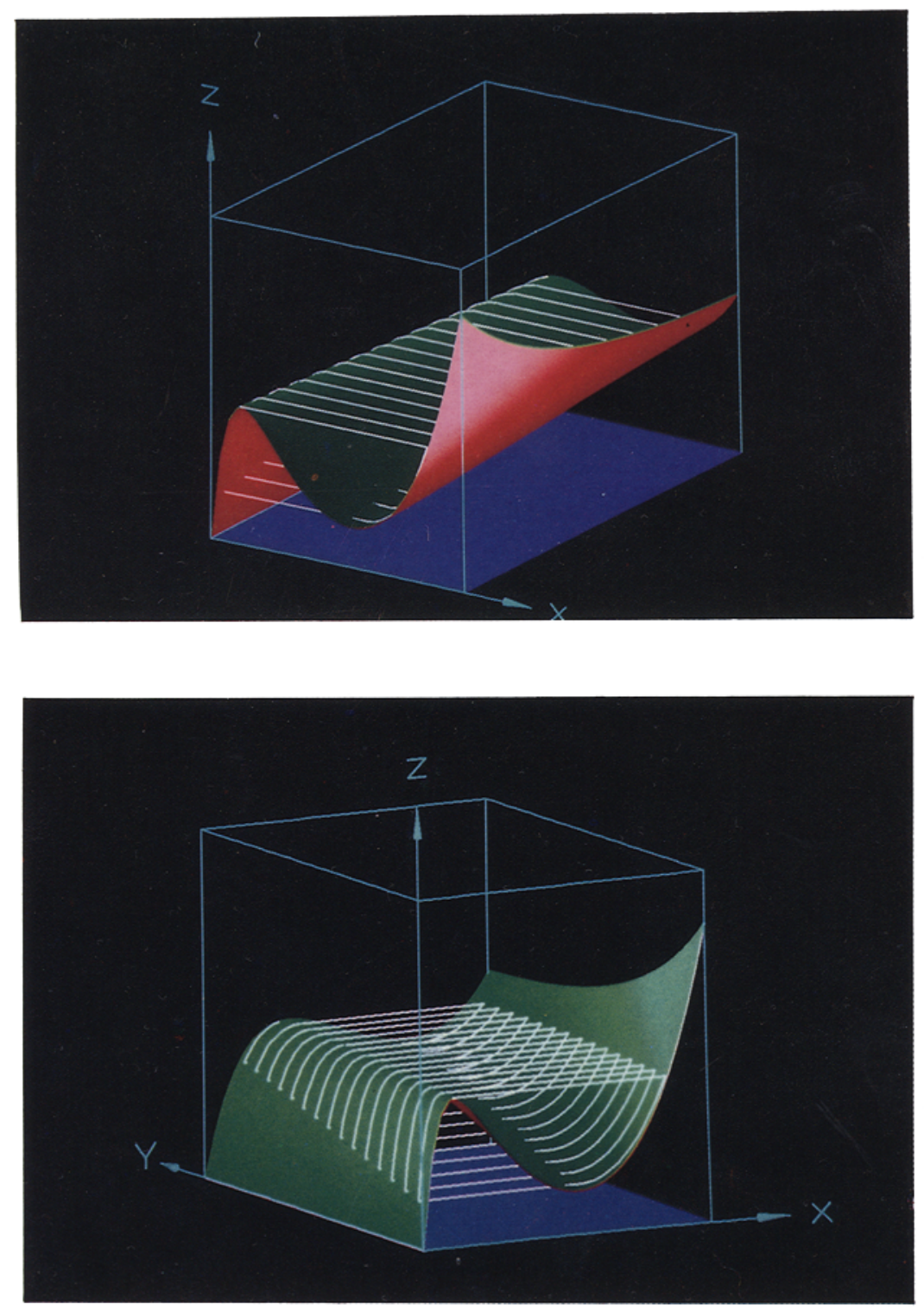

Figure 2. As the material is strained, the material characteristics evolve resulting in a surface, two views of which are shown. The $x$-axis corresponds to $\dot{\varepsilon}_{p}$, the $y$-axis to $\varepsilon_{p}$ and the $z$-axis to $\sigma$. If the system is biased in the negative-resistance region, a looping trajectory results leading to serrations.

material, that is if temperature effects are important. The equations for $\dot{\sigma}$ and $\dot{T}$ would naturally be coupled. By an appropriate analysis of this trio of equations, the required stress-strain curve (i.e. the $\sigma$ versus, $\varepsilon$ curve, where $\varepsilon$ is the total strain) can in principle be deduced. 
The models for the physical processes contributing to the yield mainly enter through the equations for $\sigma$ and $T$ (which in turn may require supplementary equations of their own). In the present work we shall ignore thermal effects, leaving us therefore with just an equation for $\sigma$. One form commonly used for the latter is (e.g. Kubin and Estrin 1985)

$$
\dot{\sigma}=h\left(\varepsilon_{p}\right) \dot{\varepsilon}_{p}+\frac{S\left(\dot{\varepsilon}_{p}\right)}{\dot{\varepsilon}_{p}} . \ddot{\varepsilon}_{p} .
$$

Here $h$ denotes the strain hardening coefficient which in general could be straindependent. The quantity $S$ defined by

$$
S=\dot{\varepsilon}_{p}\left(\frac{\mathrm{d} F\left(\dot{\varepsilon}_{p}\right)}{\mathrm{d} \dot{\varepsilon}_{p}}\right)
$$

denotes strain rate sensitivity of flow stress. $F\left(\dot{\varepsilon}_{p}\right)$ is essentially the $\sigma$ versus $\dot{\varepsilon}_{p}$ curve (at constant $\varepsilon_{p}$ ) whence a negative slope of the $F\left(\dot{\varepsilon}_{p}\right)$ curve implies also a negative value of $S$ (at a fixed $\varepsilon_{p}$ ). Thus it is that some authors associate serrated yielding with negative values for the strain rate sensitivity.

As is well-known, the microscopic mechanism for serrated yielding involves a dynamic interaction between mobile dislocations on the one hand and diffusing point defects on the other (Cottrell 1953; Freidel 1964; Beukel 1975; Kocks 1981). Our modelling does not go down to such detailed levels. Rather, we deal with macroscopic variables alone but clearly aspects of microscopic mechanisms must be reflected in the various quantities introduced above. For example, the curve for $F\left(\dot{\varepsilon}_{p}\right)$ for which we have assumed the generic form in figure 1 results from an interplay of different types of frictional forces experienced by mobile dislocations, and is the outcome of an analysis of the underlying microscopic processes (e.g. Blanc and Strudel 1987). Similarly, the strain dependence of the strain hardening $h$ depends on the mechanics of dislocation density growth and decay (e.g. Bergstrom and Roberts 1971).

As already remarked, the combination of the machine equation with the material equation (supplemented by others which characterize some of the quantities entering in the latter equation), is too involved to permit a ready and elegant analytical solution. Thus hitherto only a limited analysis has been performed, mainly to highlight aspects of the yield phenomena. Penning (1972), for example, demonstrated how the occurrence of the Portevin-Le Chatelier effect could be readily explained in terms of the negative values of the strain rate hardening over some ranges. Kubin et al (1982) have carried out a stability analysis to examine the onset of instability and the occurrence of regular serrations at low temperatures. If, however, detailed results concerning the yielding are desired, then numerical analysis or simulation is the only recourse to be had.

We now present the equations we have used and the simulation scheme we have adopted. The machine equation (1) is of course mandatory. The material part is handled by us as follows: Normally this is expressed in the form

$$
\sigma=\sigma\left(\varepsilon_{p}, \dot{\varepsilon}_{p} \ldots\right)
$$

Equation (4) must, of course, be supplemented with other information like, for example, whether $\sigma$ is separable in $\varepsilon_{p}$ and $\dot{\varepsilon}_{p}$.

We do not quite follow this approach. Instead we invert the process and consider 
the dislocation velocity $v$ as a function $f(\sigma)$ of the stress $\sigma$. Thus we write

$$
v=f(\sigma),
$$

the form of $f$ having the three-segment structure in figure 1 i.e. with a negative slope region (- see also figure 3). To accommodate the effects of work hardening which up to now we have ignored, we observe that $\sigma$ must be replaced by an effective stress $\sigma^{*}$ (Nabarro et al 1964) given by

$$
\sigma^{*}=\sigma-\alpha\left(\varepsilon_{p}\right)^{m}
$$

where $\alpha$ is the coefficient of work hardening and $m$ a parameter with value $\sim 0.5$. While the model we have chosen for representing work hardening could conceivably be bettered, we have not attempted such a step since our global objective is mainly to demonstrate how a multiplicity of factors with a complex interplay amongst themselves could successfully be interleaved in a simulation scheme, especially against a stochastic background (as we shall explain presently).

What is the state of plastic strain in the system? This we assume is well described by the Orowan equation

$$
\varepsilon_{p}=g b \rho v
$$

where $b$ is the Burger's vector, $\rho$ the (mobile) dislocation density and $g$ a suitable parameter. There remains now the task of describing the evolution of $\rho$. Here we suppose (see Bergstrom and Roberts 1971) that dislocation population dynamics is governed by a complex array of factors involving not only annihilation but also unlocking as well as immobilization. Accordingly we write

$$
\mathrm{d} \rho\left(\varepsilon_{p}\right) / \mathrm{d} \varepsilon_{p}=\Omega\left\{\rho f-\rho\left(\varepsilon_{p}\right)\right\}-\chi\left[\rho\left(\varepsilon_{p}\right)\right]^{2} \quad \rho(0)=\rho_{i} .
$$

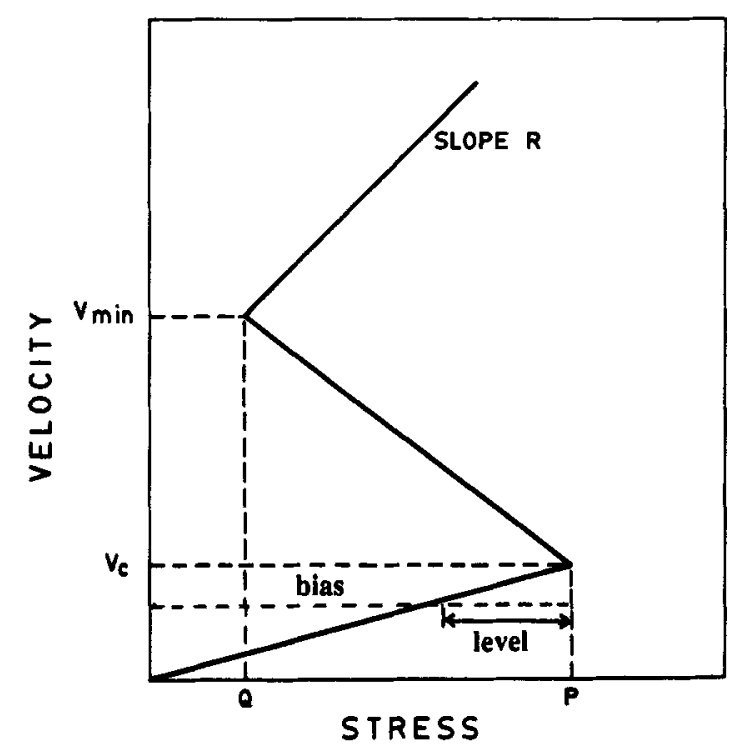

Figure 3. Three-piece approximation for $v=f(\sigma)$ used in the simulation. If the bias is just below the threshold for instability (i.e. $v_{c}$ ), then noise can take it above the threshold and cause a sudden yield drop. The barrier to be surmounted is referred to as the level. 
Here $\rho_{i}$ and $\rho_{f}$ are the initial and final densities, and $\Omega$ a constant related to the remobilization rate. While the first term on the r.h.s. is based on the model of Bergstrom and Roberts (1971), the second term has been included on the recommendation of Nabarro (private communication; see also Nabarro et al (1964)). Once again we add the caveat that no claim is being made as to the use of the best possible model as regards the evolution of dislocation population. We believe that for demonstrating the objectives we have in mind, the model proposed is quite adequate.

So far, all the equations considered are strictly deterministic. Thus it is natural to expect at best only periodically spaced yield drops, an anticipation which is corroborated by the earlier findings of Kubin et al (1982) and of Neelakantan and Venkataraman (1983), where too a deterministic approach was employed and only regular serrations were obtained. On the other hand, it is a fact of life that serrations observed in laboratory experiments are often quite irregular both in terms of their spacings as well as their amplitudes, strongly suggesting the presence of underlying stochastic effects. Nevertheless, the role of stochastic processes in metallurgical phenomena seems to have received scant attention. (An exception is the role of fluctuations in anelastic relaxation, investigated by Balakrishnan et al 1978; see also Venkataraman 1982).

Starting with the classic work of Einstein on Brownian motion, the role of noise in physical processes has been extensively studied both theoretically and experimentally. (For an elegant comprehensive introduction to noise in physical systems, see MacDonald 1962.) In the ultimate analysis, noise such as we are interested in arises due to random thermal motions of the atoms. However, considering that we are operating at the level of macroscopic variables, it is reasonable to suppose that the medium acts as a heat bath, producing random effects in the variables of interest. This is not an unreasonable supposition and indeed in the classic analysis of Einstein alluded to, the topic of investigation was the random motion of a micron-size object namely a pollen grain, in the heat bath provided by the atoms. It is pertinent to note that compared to atoms, the pollen grain itself is "macroscopic" in size. Nevertheless, due to the effects produced by the heat bath, it (the Brownian particle) executes random motion. In the same spirit, we suppose that the heat bath (provided by the atoms) generates stochastic effects.

The question now arises as to which variables we should consider as being stochastic in character. The choice would depend upon what we consider to be the "primary" variable(s). While many we have discussed with have suggested various choices, our own preference has been to treat velocity as the random variable. In microscopic theories where one analyses the frictional forces opposing dislocation motion, one considers a single dislocation. Plastic flow, on the other hand, is due to the collective motion of a large number of dislocations. Thus, the quantity $v$ in equation (7) really refers to the velocity of group of dislocations.

In our discussion thus far, we have implicitly treated $v$ to be a deterministic quantity. On the other hand, Einstein's analysis suggests that it would be fruitful to regard $v$ as a stochastic variable. Indeed, if one considers the progress of a convoy of vehicles, the velocity of the convoy may be expected to fluctuate due to various obstacles like traffic lights, bad roads etc. Thus the choice of $v$ as a stochastic variable is quite reasonable.

Having made this decision, we now need a quantitative prescription. In the Lan- 
gevin-type analysis of Brownian motion, one takes

$$
v=\langle v\rangle+\eta,
$$

where $\langle v\rangle$ is the average value and $\eta$ a fluctuating quantity. We prefer instead the form

$$
v(\sigma)=\langle v(\sigma)\rangle+\beta(\sigma) \eta .
$$

Compared to the Langevin form, the noise term proposed by us depends on the state of stress through the quantity $\beta(\sigma)$. Our noise term is thus multiplicative (Schenzle and Brand 1979). The physical reason for our suggesting multiplicative noise is based on analogy with noise effects in a diode. To appreciate this analogy, we first observe that the Orowan equation (7) has the same form and the same physical content as the familiar conductivity equation $j=n e v$ where $j$ is the current per unit area, $n$ the electron density, $e$ the electronic charge and $v$ the velocity. The shot noise in a diode increases multiplicatively (Ziel 1970), and it is this which prompted us to adopt a similar model.

One could argue that as in all birth and death processes, the dislocation density too could be regarded as a stochastic variable. Very true, and indeed if it is so regarded it might be reasonable to suppose that it also is represented by multiplicative noise. The question now arises whether both $v$ and $\rho$ are independently random or whether random fluctuations in one influences the other. This is a tricky question which we have skirted by simply treating $v$ alone as having the form in (10) and letting all other quantities "slave" $v$ as far as fluctuations are concerned.

We now turn to the simulation process which, as mentioned earlier, is a convenient line of attack when one has to deal with complicated and coupled evolution equations. Time marching done in a computer enables one to have a "blow by blow" account of the evolution process, and by comparing simulation results with those of laboratory experiments, one has the possibility of progressively refining the models entering the simulation. For this, clearly simulation and laboratory experiments must go hand in hand and indeed through this simulation can even eventually acquire considerable predictive power, as has happened for example in laser fusion, in aerodynamic design and in automobile accident analysis.

Our simulation scheme may be briefly described as follows: The process starts with a specification of the values of the various parameters, as also the initial conditions, comprising the values of $\varepsilon_{p}(0), \sigma(0)$ (which is normally taken as zero) and $\rho(0)$ (which is set equal to the value of $\rho i$ assumed).

Time is marched in equal steps $\Delta t$, and at any given time $t$, the following computations are performed:

(i) $\rho(t)$ is computed using equation (8) and the values of $\rho(t-\Delta t), \varepsilon_{p}(t-\Delta t$.$) and$ $\varepsilon_{p}(t)$ which itself is obtained from

$$
\varepsilon_{p}(t)=\varepsilon_{p}(t-\Delta t)+\dot{\varepsilon}_{p}(t-\Delta t) . \Delta t .
$$

(ii) $\langle v(t)\rangle$ is computed from equations (5) and (6) using $\sigma(t-\Delta t)$ and $\varepsilon_{p}(t-\Delta t)$, and the approximate three-piece curve in figure 3.

(iii) The velocity is now randomized according to equation (10) to obtain $v(t)$. 
(iv) $\sigma(t)$ is computed according to

$$
\begin{aligned}
\sigma(t) & =\sigma(t-\Delta t)+\dot{\sigma}(t-\Delta t) \cdot \Delta t \\
& =\sigma(t-\Delta t)+\left[\dot{\varepsilon}_{a}-\dot{\varepsilon}_{p}(t-\Delta t)\right] \cdot K_{\mathrm{eff}} \cdot \Delta t
\end{aligned}
$$

(v) $\dot{\varepsilon}_{p}(t)$ is computed using $\rho(t)$ and $v(t)$ in Orowan's equation.

All quantities for time $t$ are now determined as also the inputs needed for calculations at time $(t+\Delta t)$. Some additional remarks are made in the Appendix.

The simulation scheme described above pertains to the case of homogeneous yielding. The scheme for inhomogeneous yielding is similar and will be described in a later section.

Based on the above scheme, simulations results for a wide variety of cases have been obtained. The range of parameters explored is discussed in the Appendix. It is to be noted that for numerical convenience, scaled parameters were employed in the actual simulation. It is also worth emphasizing that the fact that we have not made explicit use of equation (2) does not imply that quantities like strain hardening and strain-rate hardening do not enter into the simulation. In our scheme they are implicit parameters but if so desired, the simulation scheme could be altered to make the role of these widely used parameters more explicit. The approach adopted here (especially the use of a three-piece curve as in figure 3) seemed simpler in the context of the extensive survey of the simulation possibilities we were interested in.

\section{Results obtained with a homogeneous yielding model}

We shall now present some of the results obtained using the model and the simulation scheme described in the previous section. As a prelude, we present in figure 4 a global overview of the widely different kinds of patterns we have been able to simulate. The nomenclature used follows that of Pink and Grinberg (1981). While the model of homogeneous yielding might be viewed with suspicion, it is nevertheless noteworthy that we have been able to mimic realistic patterns.

\subsection{Role of noise}

As pointed out earlier, an important aspect of our work is the explicit consideration (for the first time) of noise in relation to yielding. It seems useful therefore to understand the role played by noise in influencing the yield curves. This is illustrated in figure 5 . On the extreme left are two patterns, one of which (5a) shows regular serrations of the B-type while the other $(5 \mathrm{~d})$ shows no serrations. The patterns to the right of the above two illustrate the modifications brought about by the inclusion of the noise effects. In the case of the pattern with type-B serrations, noise does not seriously affect the spacings between adjacent drops although the amplitudes of the pulses show slight fluctuations. In the other case, the previously smooth curve is now disturbed by the appearance of spikes; the greater the noise, the larger the number of spikes. It may also be observed that both the spacings and the amplitudes of the spikes fluctuate.

The occurrence of the spikes is easy to understand with the help of figure 3 . We have here a situation where the bias curve or the load line intersects the 


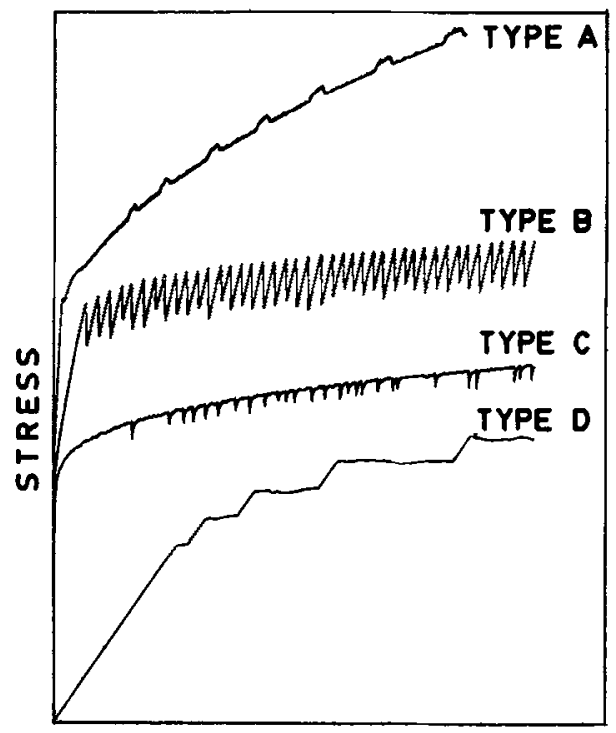

STRAIN

Figure 4. Various types of serrations obtained by simulation. The shapes of the curves obtained compare well with the archetypes described by Pink and Grinberg (1981) and by Rodriguez (1984).
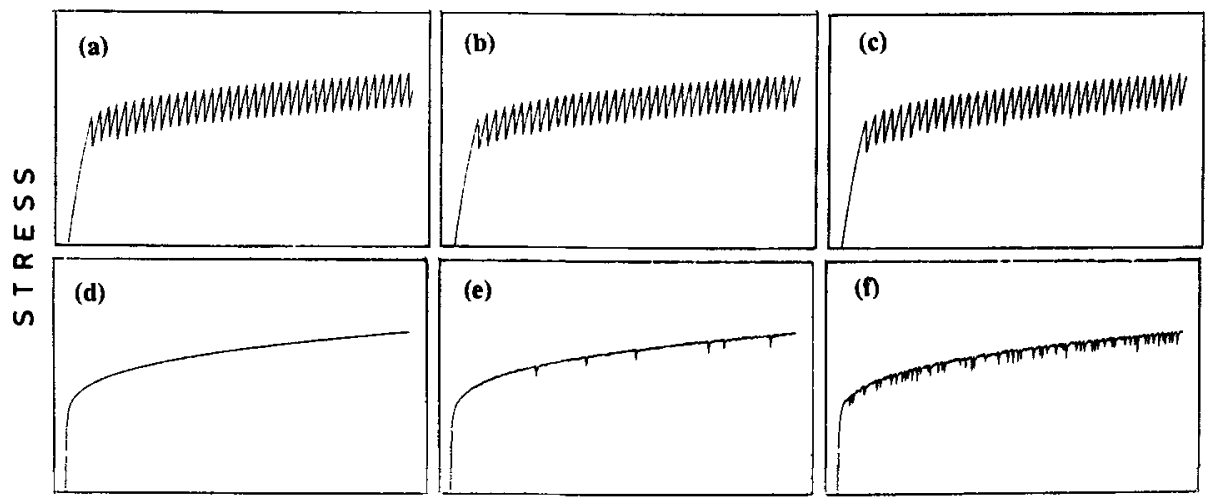

ST RAIN

Figure 5. Simulated curves to demonstrate the effects of noise on types $B$ and $C$ serrations. 5(a) and 5(d) refer to yield in the absence of noise. The curves in the middle and the right are with moderate and high noise amplitudes respectively.

characteristic curve in a normally stable region. If there were no noise effects associated with the velocity, the yield curve would be smooth, as indeed is observed in figure $5(\mathrm{~d})$. But on account of the randomization of the velocity, occasionally the operating point might make an excursion into the unstable region, resulting in a sudden stress spike or yield drop. From this it is natural to expect that the greater the amplitude of the noise affecting the dislocation velocity, the more frequently would the spikes appear. This expectation is borne out both by figures $5(\mathrm{e})$ and $5(\mathrm{f})$. 


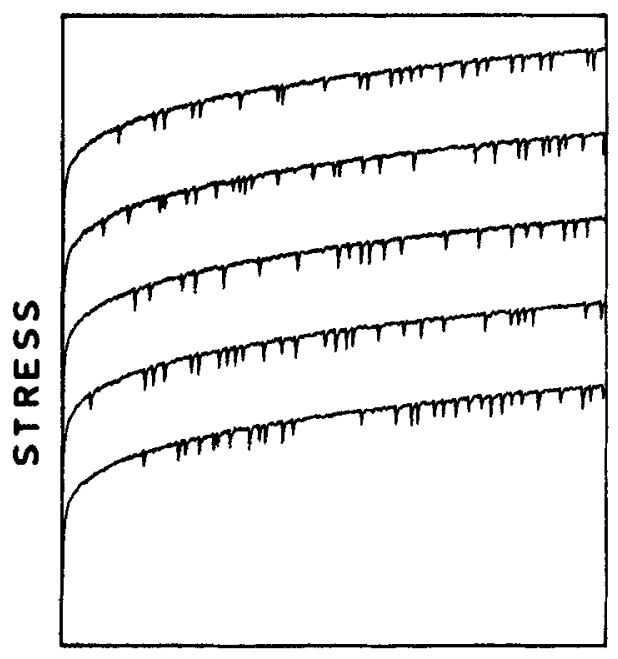

STRAIN

Figure 6. Several reruns of a yield curve. Though the parameters used were the same, the occurrence of the spikes varied from run to run as is to be expected since they are noise triggered.

The random nature of the spike sequence is further highlighted in figure 6 which shows several reruns under the same conditions. Spikes appear in every pattern but in different ways.

A few remarks now about the statistical distribution of the yield drops in figure 6 . Caution is needed in carrying out a statistical analysis for, thanks to work hardening, we are dealing with a nonstationary situation while riding a yield curve. However, even though one may have lack of stationarity over extended periods of time, one could divide the time elapsed into small and convenient intervals $\Delta t$, assume that processes are stationary during each of these intervals, do numerous reruns as in figure 6, accumulate sufficient data for all the successive time slices and then analyse the data for each time slice separately. Indeed, an experimentalist working with real samples would have to go through such a tedious process if he wishes to overcome difficulties associated with lack of stationarity. (For him, equal time slice would translate into equal strain slices since total strain is proportional to elapsed time.) For us on the other hand there is the convenient alternative of simply switching off the work-hardening term. Once this is done, there are no longer any problems with lack of stationarity. Data obtained in this fashion are presented in figure 7.

As earlier remarked, spikes appear in figure 6 since the biasing is close to the threshold for instability. Some of our results can therefore be understood in terms of the level-crossing problem (Papoulis 1965). The meaning of the level to be crossed can be found in figure 3, and the question is how often is this level crossed. Analytical results for this problem are available, and are compared with our results in figure 8. While there is overall agreement, there is also a systematic deviation at higher noise levels. This merits further investigation.

In passing it must be mentioned that in our earlier paper we had speculated on the possibility of chaos contributing to the observed irregularities in yielding. In 


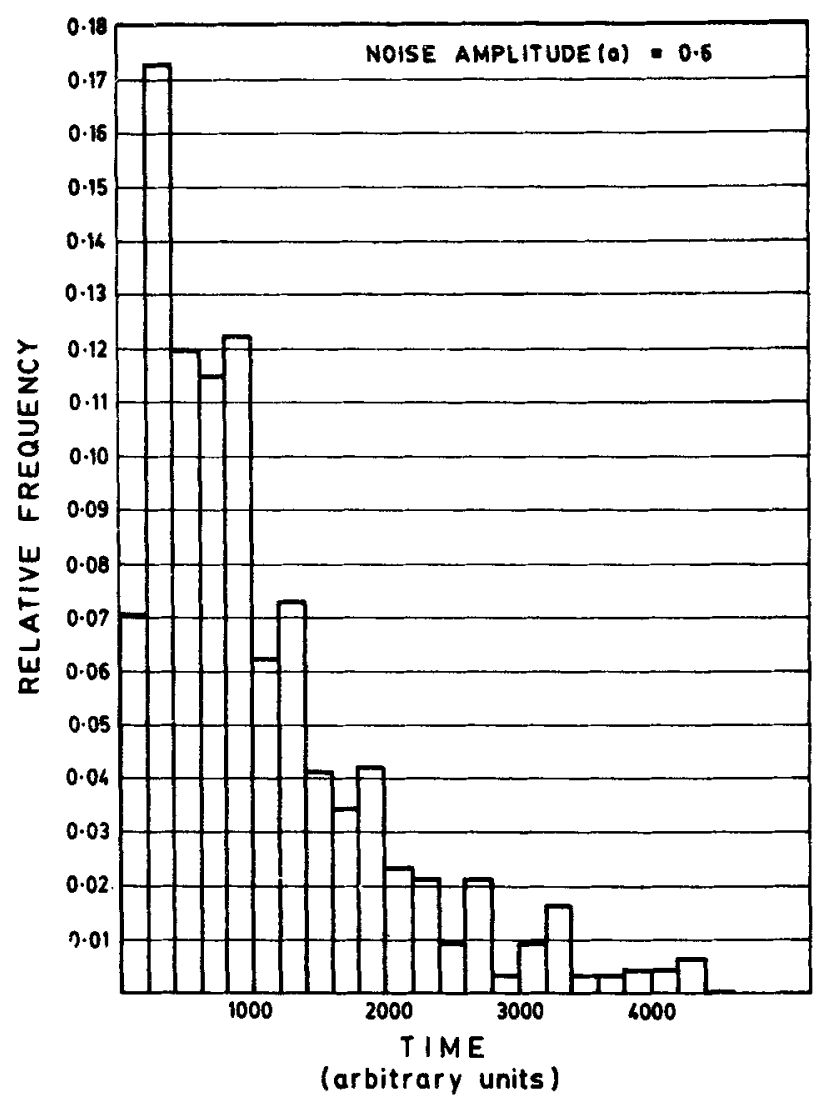

Figure 7. Histogram of the time separation between adjacent spikes, corresponding to a fixed noise amplitude.

fact, Ananthakrishna and Valsakumar (1983) discussed an explicit model based on chaos. In the present work we have not found it necessary to invoke effects due to chaos.

\subsection{Temperature dependence}

Figure 9 shows a family of curves representing a situation where the strain rate is kept the same but the material is held at different temperatures in the different tests. As far as our simulation is concerned, this effectively implies varying suitably the quantities $v_{c}$ and $v_{\min }$ indicated in figure 3 (see also figure 8 of Neelakantan and Venkataraman 1983). Also presented in figure 9 are yieid curves at various temperatures for an Al-Mg alloy, as obtained by Hall (1970). The similarity between the results oî our simulation and those obtained in laboratory experiments is striking. In presenting this comparison, we emphasize that our effort is directed mainly towards demonstrating how the sequence of patterns observed in experiments can be mimicked, especially in relation to the noise effects. Without noise, spikes do not appear in the simulated yield curves. No particular significance is attached by us at present to the parameter values used in the simulation vis-a-vis 


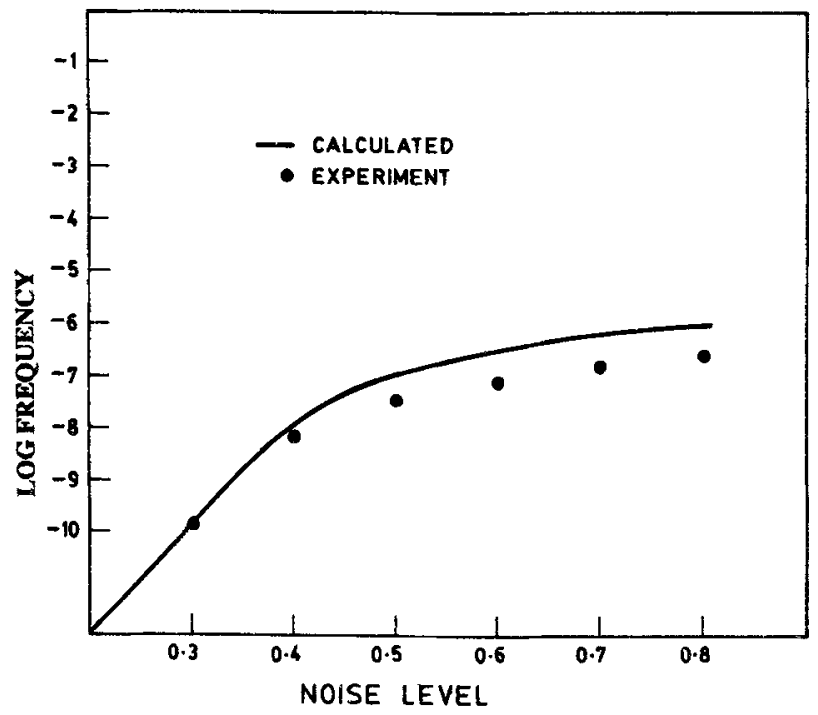

Figure 8. The observed level crossing rate as a function of noise amplitude, compared with theoretical values.
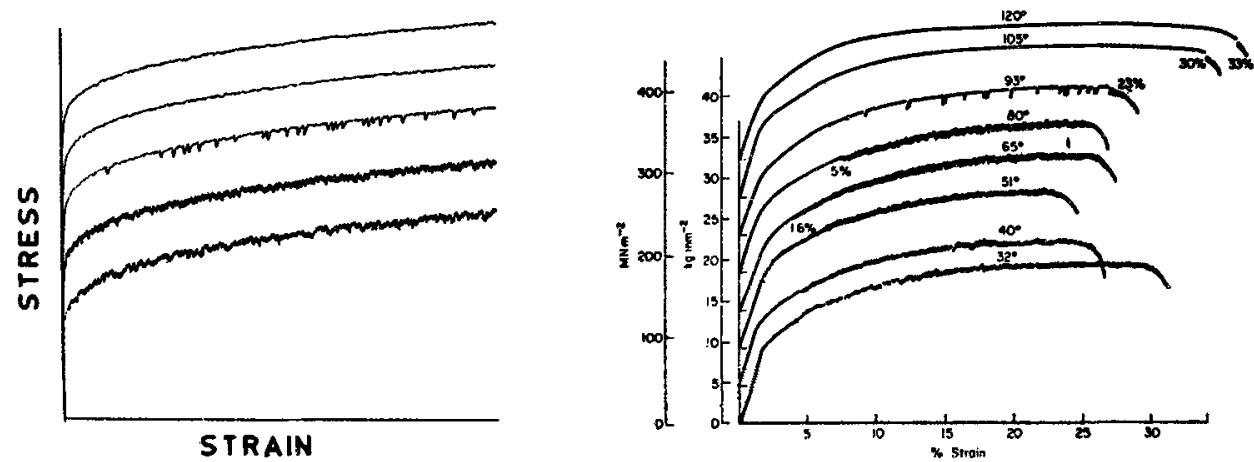

Figure 9. On the left are yield curves obtained by simulation (with a suitable choice of parameters), and with $f\left(\sigma^{*}\right)$ in equations (5) and (6) varied as described by Neelakantan and Venkataraman (1983), in order to mimic temperature variation. On the right are the results for an Al-Mg alloy as reported by $\mathrm{Hall}$ (1970).

those actually pertinent to $\mathrm{Al}-\mathrm{Mg}$, especially because we have ignored effects of inhomogeneity. At the same time it is evident how simulation results could be made to approach closely those obtained in actual experiments. It is this which prompts the comment that greater emphasis on modelling and simulation coupled with comparison with experiments could lead to a better understanding of the mechanics of the yielding process.

\subsection{Instrument resolution and pulse shape}

It is our view that apart from distribution, the shape of the yield pulse also contains 

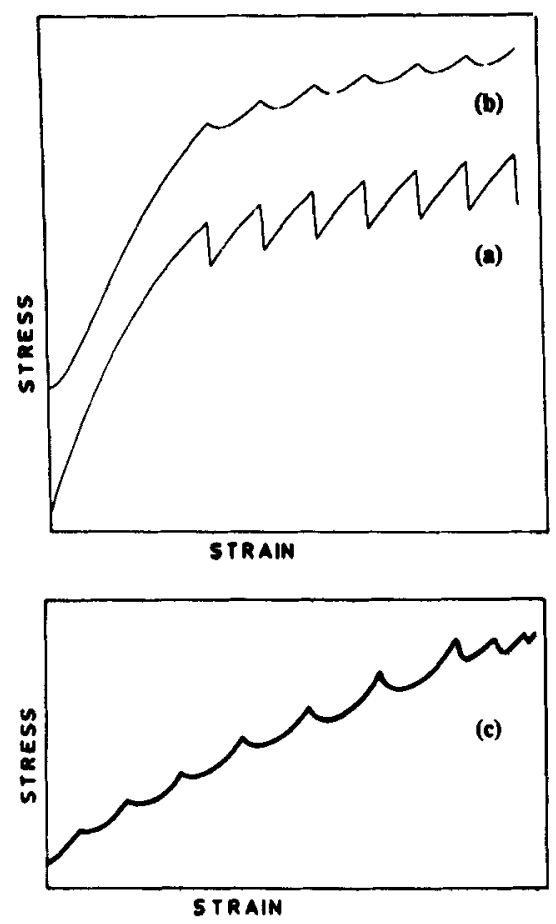

Figure 10. Effect of instrument time constant on the shape of the yield drop pulses. The curve (a) is a simulated curve corresponding to a load cell with infinite resolution. The curve (b) shows the appearance due to finite instrument resolution. Presented for comparison is (c) which shows a portion of the experimental yield curve for stainless steel (after Rodriguez 1984).

information. If the shape becomes the object of study, then due consideration must be given to the instrument response time. Figure 10 gives an example of how response time can alter the observed yield curves. Generally speaking, when strain gauges are used as transducers for sensing the load, one has a bandwidth of only around $100 \mathrm{~Hz}$. Capacitative transducers offer a much higher bandwidth $(\sim 1-$ $10 \mathrm{kHz}$ ) and therefore permit a more careful study of pulse shape (Schwarz and Funk 1985).

\subsection{Effect of interruption}

The effect of interrupting the testing has been investigated, and a typical outcome is illustrated in figure 11. It is gratifying that this curve is strikingly similar to what is reported in the literature (e.g. Lucke and Mecking 1973).

\section{Inhomogeneous yielding}

\subsection{A preliminary overview}

We now relax the rather drastic assumption made in the previous section, namely 


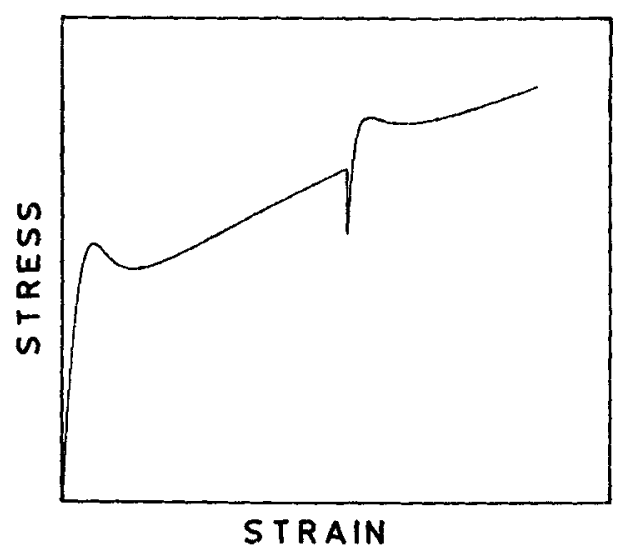

Figure 11. Simulation of the effect of strain ageing. The applied strain rate was made zero and then reapplied after some time.

that the yielding process is homogeneous. While the assumption of homogeneity might not be objectionable as long as there are no serrations, there is wide unanimity that serrations are a clear indication of inhomogeneous yielding. Starting with the work of Penning (1972), there have been many attempts at mathematical modelling and analysis but really speaking, none of these have progressed deep enough. The principal points which have emerged so far are the following:

(i) The specimen may be regarded as being made up of contiguous domains.

(ii) The strain is not uniform throughout, and at any given instant yielding occurs in that domain where the strain is the least.

(iii) Deformation propagates across the sample, generally sweeping from one end to the other.

(iv) Deformation bands which propagate are broadly of two types (a) hopping bands and (b) continuously moving band.

(v) Homogeneous yielding becomes inhomogeneous when the former process becomes unstable.

We shall now consider some qualitative aspects of the above, so that a better appreciation may be had of our work (to be described). The discussion is best done with the help of some three-dimensional plots. For convenience, we shall ignore stochastic effects for the present.

We consider first the case of hopping bands. In figure 12 is shown the surface corresponding to the mechanical equation of state. We assume, as does Penning (1972), that there is present in the material a uniform strain gradient characterized by a slope $m(=\delta \varepsilon / \delta x)$. We now examine the processes happening in the material at some particular instant of time. As far as the flow stress is concerned, it is equilibrated across the length of the sample with a velocity close to that of sound (Kubin and Estrin 1985). Thanks to this, yield now occurs in that region of the sample where the strain is the least. This region is indicated on the left half of figure. If $d$ is the width of the region and if $f=(d / L)$ where $L$ is the length of the sample, $\dot{\varepsilon}_{o}$ the effective applied strain rate in the small slice is given by

$$
\dot{\varepsilon}_{o}=\left(\dot{\varepsilon}_{a} / f\right)
$$




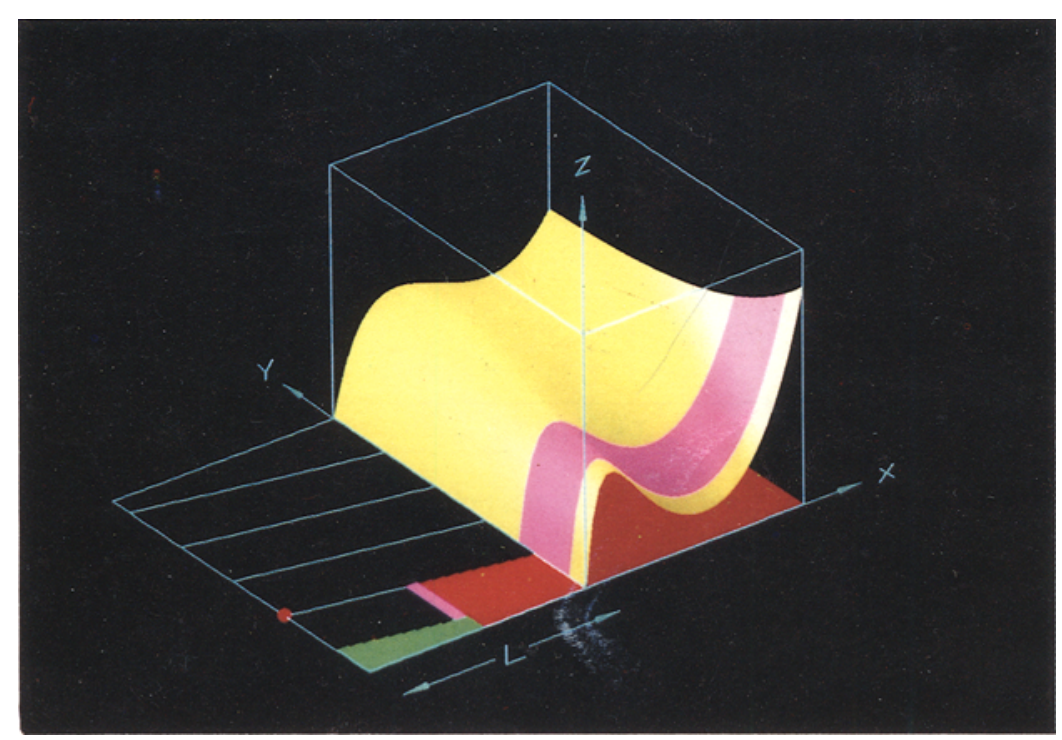

Figure 12. Shown on the right is the $\dot{\varepsilon}_{p}-\varepsilon_{p}-\sigma$ surface. The labelling of the axes is as in figure 2 . On the left is shown the strain state in the various domains across the sample-at a particular instant $t$ say. The red portion represents yield that has already occurred, while the green portion shows the strain in the region where the strain front has yet to propagate. The region shown in magenta represents the strain jump that would occur in the next time step $\Delta t$. The region of the $\dot{\varepsilon}_{p}-\varepsilon_{p}-\sigma$ surface called into play during this jump is shown by the same colour. The strain front propagates across the sample, causing such jumps successively in the various domains. Notice the front climbs a hill. When the end point shown by the red dot is reached, a new strain front is initiated and propagates across the sample. The lines in cyan represent the various subsequent sweeps of the strain front.

What happens in this region now under consideration is dictated by (i) the nature of terrain corresponding to the slice shown in magentai in figure 12 and (ii) the bias $\dot{\varepsilon}_{0}$. If the latter is in the stable region. then the stress increases smoothly, even as the strain in the region increases bs an amount $\Delta k$. If the bias $\dot{\varepsilon}_{n}$ is in the unstable region. the increase of strain 1 : is accompanied by a yield pulse, precisely as described in $\$ 2$. Once the strain in the domain under consideration jumps by the amount $\Delta \varepsilon$, the domain ceases to be one with the least strain. Deformation activities now shift to the adjacent domain. where a similar scenario is enacted. In this way, as the strain front climbs up the strain hill. a series of vield pulses are produced, provided of course the bias $\dot{c}_{n}$ is in the unstable region. On the other hand if $\dot{c}_{0}$ is in the stable region, the yield curve would be smooth. If $\dot{\varepsilon}_{0}$ corresponds to a case of marginal stability (rather as in figure 3). then it is easy to imagine that noise could cause yield drops to occur during the progress through some domains.

The case of the continuous band model is essentially a limiting case of the one discussed above, with the domain width $\rightarrow 0$. In this case. one can visualize the growth of strain across the sample as in figure 13. Essentially, strain fronts sweep across the sample one after the other. The velocity $r$ with which the strain front advances is determined by material processes. Attention is drawn to the fact as 


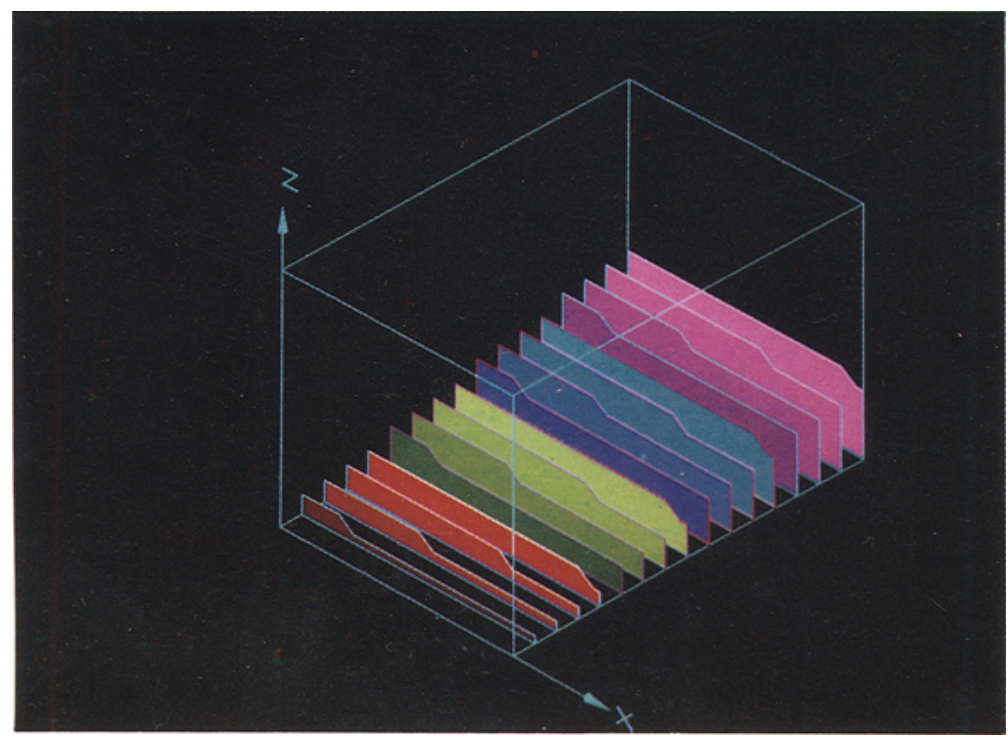

Figure 13. Here the $x$-axis denotes distance across the sample, the $y$-axis denotes time and the z-avis denotes $a_{F}$. The figure essentially summarizes the strain state across the sample at various instants of time. As can be seen. strain fronts keep sweeping across the sample; the different fronts are shown in different colours.

has also been done by Kubin and Estrin 1986) that the propagation of the strain front is analogous to that of the electric field front in the Gunn diode.

The comprehensive and pictorial overview just presented is based on remarks scattered in many places in the literature, notably the work of Penning (1972). In our view, there is one important ingredient missing while constructing pictures as above. It is nice to visualize an advancing strain front but presumably adjacent regions yield in succession because of a "flow" of mobile dislocations across the specimen i.e. essentially from domain to domain. It is natural therefore to expect something like a continuity equation, familiar in other fields where transport processes are considered (e.g. neutron diffusion). Since earlier modelling (e.g. by Penning) was restricted to the simplest possible coupling scheme linking the machine equation with the mechanical equation of state, processes like dislocation flow were not given explicit consideration, it being tacitly assumed perhaps that they would suitably influence (implicitly) the parameters entering the analysis. In a later subsection we suggest how flow could be incorporated.

\subsection{Simulations a la Penning}

Among the various studies on inhomogeneous yielding we have carried out, we report first the work done using essentially Penning's model. We assume the material is composed of $N$ identical and contiguous regions or segments, each of which behaves independently of the others. The response of the various segments is 
thus simulated essentially as described in the previous section but with the difference described below.

Consider the time step $t$, and let $\dot{\varepsilon}_{p}(j ; t)$ denote the plastic strain rate in segment $j$ at that time. The total plastic strain in the material is calculated as

$$
\dot{\varepsilon}_{p}(t)=\frac{1}{N} \sum_{j=1}^{N} \dot{\varepsilon}_{p}(j ; t) .
$$

This is then used in the machine equation to obtain $\dot{\sigma}(t)$ and from it $\sigma(t)$. Implicit in the above is the assumption that dislocations remain confined to their respective domains or segments within which, however, they may be mobile, become locked, annihilated etc.

We have explored the above model under different conditions of applied strain rate, as well as with and without noise. In all cases, a small strain hill was introduced as originally considered by Penning.

Figure 14 shows the result obtained with 8 domains, with no noise and a relatively small applied strain rate, the biasing being in the unstable region. We observe features described by Penning as the hopping band. It is interesting that there is a small "dead time" between the cessation of the strain-rate pulse in one segment and its build up in the next one. Also noteworthy is the fact that apart from the strainrate pulses (which are localized), there is a small but nonzero background yielding that goes on all the time in all the segments.

Hopping bands are produced only under conditions of instability. If the bias is

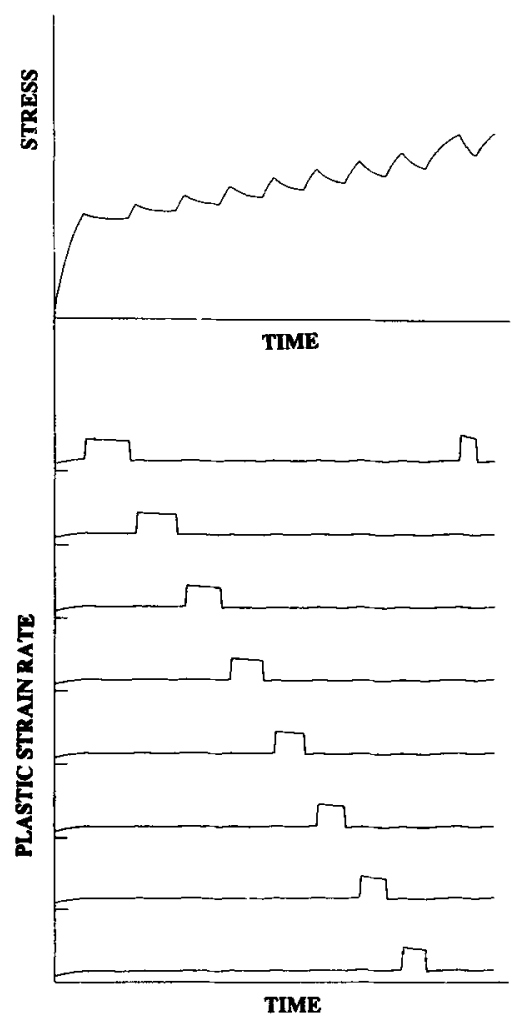

Figure 14. Simulation results as obtained with Penning's hopping model for moderately low applied bias and with no noise. Serrations are obtained as predicted by Penning, with every serration being associated with a distinct strain-rate pulse. The lower half of the figure shows the strainrate profile in different domains. The small horizontal marks at the left define the zero level for the various domains. Observe that (i) strain rate pulses occur successively in the different domains, and (ii) there is a small background, uniform straining that goes on all the time in all the domains. 


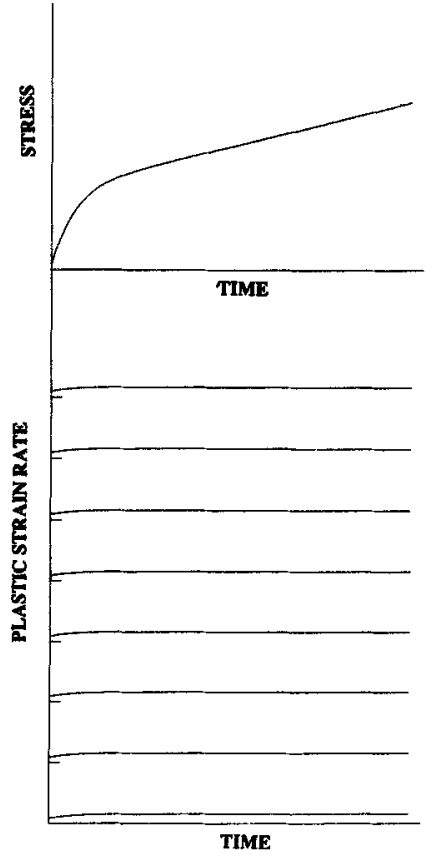

Figure 15. Simulation results for the 8-domain sample obtained with no noise, and low bias but applied in the stable region. Observe the marked contrast with the previous figure.

lowered further so as to bring the operating point to the stable region, the yield curve is smooth as figure 15 shows. On the other hand, if the system is marginally stable and noise effects are included, the yield becomes jerky as may be seen in figure 16. When the applied strain rate is high, the results obtained are as in figure 17 and correspond to the case described by Penning as continuously moving bands. Strain rate pulses occur rather as envisaged by him.

\subsection{Models with dislocation flow}

The simulations described in the previous subsection are in the spirit of Penning's earlier work which, as already pointed out does not give any explicit consideration to the flow of dislocations across the sample. We have therefore attempted a model which incorporates, to some extent at least, the notion of such a flow.

Once again we regard the material to be divided into segments, but this time is not necessarily identical. They could, for example, represent grains of various sizes. At the start, we specify the density of dislocations in each region and also assign a weight factor representative of the size of the region.

Consider a particular domain $j$ at time $t$. Yield in this domain is computed exactly as described in $\S 4$ with one difference, namely that to the density $\rho(j ; t)$ computed using equation (8), we add the quantity $f . \rho(j-1 ; t-\Delta t)$ where $f$ is a suitable fraction. Thus the density in $j$ is

$$
\rho(j ; t)+f \rho(j-1 ; t-\Delta t)=\rho^{*}(j ; t) \text { say. }
$$

The second term on the left side mimics inflow. Naturally, once this flow is allowed for, there must be a corresponding reduction of density in the segment $(j-1)$.

The quantity $\rho^{*}(j ; t)$ is now used in Orowan's equation to compute $\dot{\varepsilon}_{p}(j ; t)$. The 


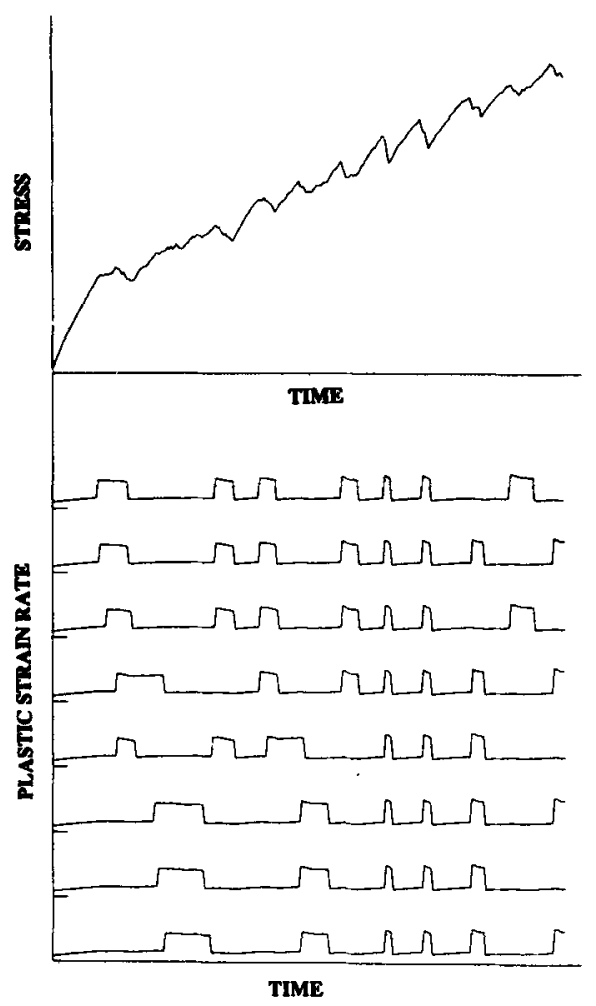

Figure 16. This shows the effect of adding noise to the experiment of the previous figure. Jerky flow is now the result.

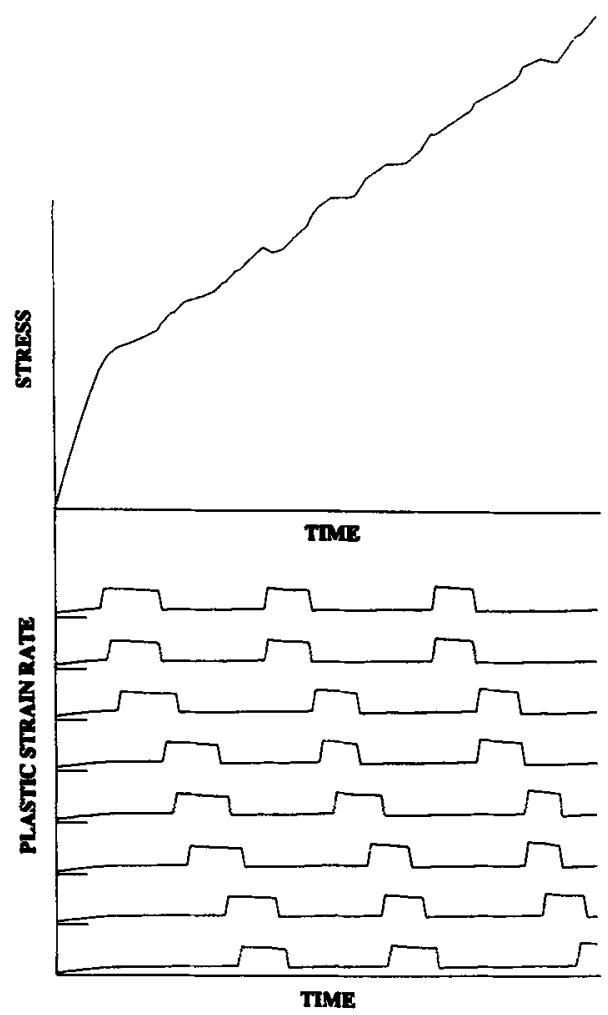

Figure 17. One example of a simulation which roughly corresponds to Penning's continuously moving band case. In contrast to the results of figure 14 , high yielding occurs simultaneously in several domains. This broad region of yielding spreads across the sample leading to a front as illustrated in tigure 13. 
overall strain rate $\dot{\varepsilon}_{p}(t)$ is obtained by taking a weighted average of the $\dot{\varepsilon}_{p}(j ; t)$ 's i.e.

$$
\dot{\varepsilon}_{p}(t)=\frac{1}{N} \sum_{j=1}^{N} w(j) \dot{\varepsilon}_{p}(j ; t),
$$

where $w(j)$ is the weight factor. This value of $\dot{\varepsilon}_{p}(t)$ is then used for calculating $\sigma$, as described earlier.

The model crucially depends on the initial distribution of the dislocation density and the weightages given. The yielding of any one region does not necessarily continue indefinitely. It ends when a sufficient number of dislocations have been swept away into the next domain, and the remnant dislocations become immobilized.

We now present a few sample results obtained with this model. Figure 18 shows two yield curves both obtained with a 12-segment model with equal weightage for the sections. However, there was a small gradient in the initial dislocation density, it being the highest in the first segment. In (a) the applied bias was in the unstable region whereas in (b) it was in the stable region. In the latter case there are no serrations and in fact, the final plastic strain acquired by the different segments is the same which suggests homogeneous yielding, effectively. On the other hand, when the strain rate is somewhat higher there are not only serrations but, further, the strain acquired by the different segments also fluctuates. Clearly in this case the yielding is truly inhomogeneous.

Figure 19 presents another result obtained by us, clarifying further aspects of inhomogeneous yielding. In figure 19 (a) is shown the yield curve while in 19 (b) are plotted the plastic strain rates in the different segments as a function of time.

Figure 20 shows a comparison with results obtained by Yoshinaga et al (1971) for
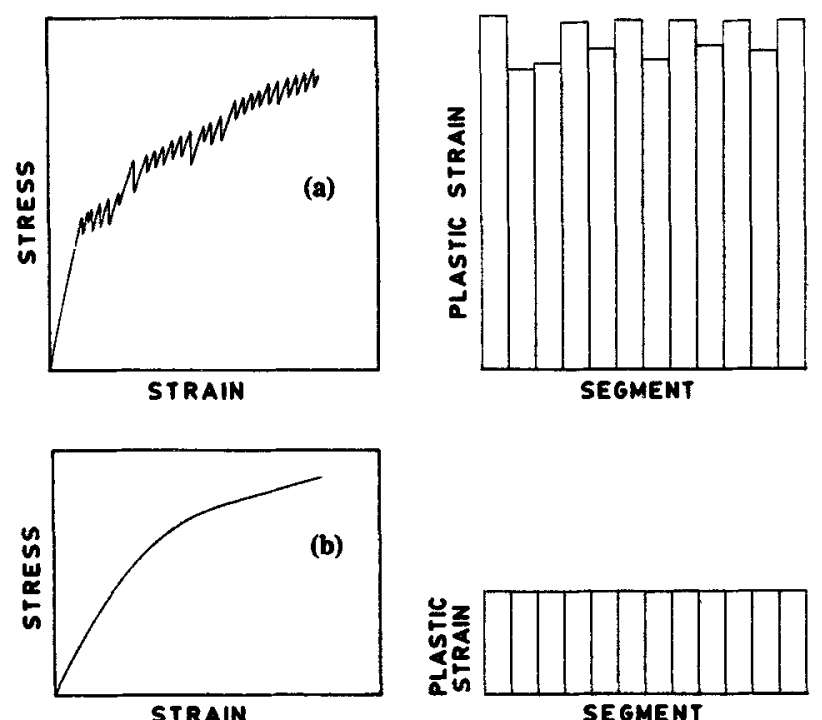

Figure 18. Yield curves obtained with a model simulating inhomogeneous yielding but at different strain rates. The upper curve (a) is for an applied strain rate in the unstable region while the lower curve (b) applies when the bias is in the stable region. Shown alongside the yield curves are the plastic strains in the various segments, at the end of the experiment. 


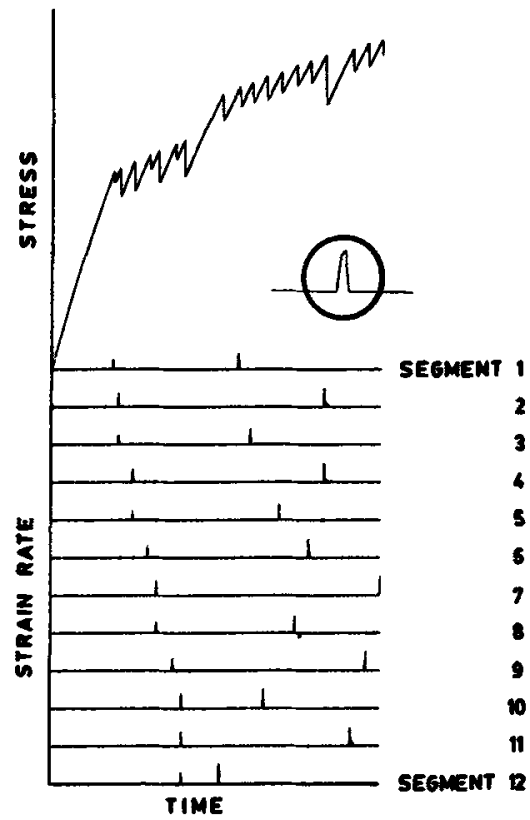

Figure 19. Shown here is the yield curve for a 12-segment model and the plastic strain rates in the various regions. The inset depicts the shape of the plastic strain-rate pulse.
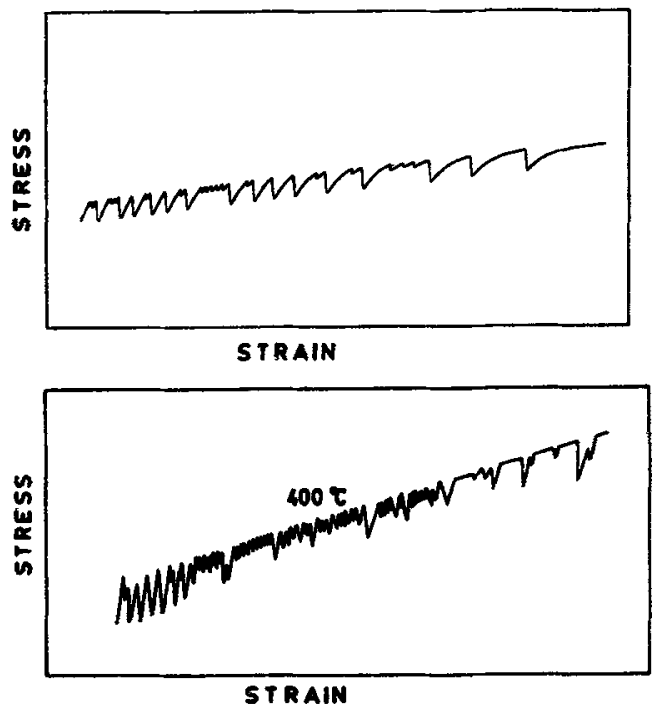

Figure 20. On the top is a portion of a simulated curve using the inhomogeneous model. The lower curve is a section of the yield curve observed by Yoshinaga et al (1971).

vanadium. The effect of suddenly varying the strain rate is shown in figure 21 , where the results of Leslie and Cuddy (1972) also are shown for comparison. Occasionally, bunching of spikes is observed in the yield curve as may be seen in figure 22 (a) which shows some results for austenitic stainless steel (Rodriguez 1984). This may be compared with figure 22 (b) wherein we show the results of simulation 

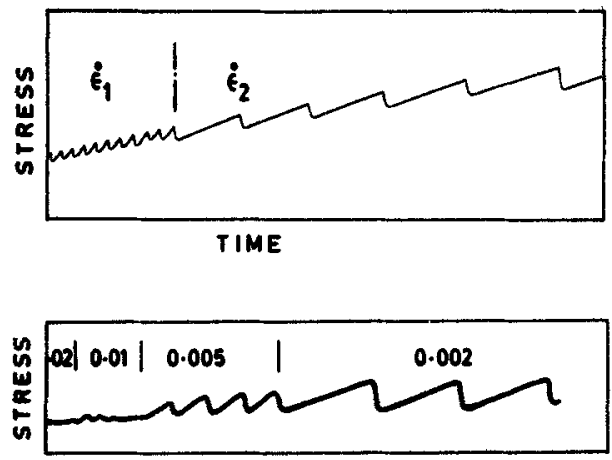

TIME

Figure 21. Effect of varying the strain rate. The simulated curve is shown at the top while at the bottom is shown the result of Leslie and Cuddy (1972).

obtained with a two-segment model, representing the presence of two types of regions with quite different grain sizes.

The results presented here are representative. Clearly, many ramifications of the above studies are possible.

\subsection{Sandpiles and yielding}

Recently, Bak et al (1988) studied a class of extended dissipative dynamical systems which evolve naturally into a critical stage referred to as the self-organized critical state (SOCS). By way of exploring the spatial and the temporal features of such systems, they have studied various models of transport. Following them, we have performed some studies on a one-dimensional sand pile as it displays features of interest to the present problem.

We consider a one-dimensional sand pile of length $N$, consisting of $N$ units. The boundary conditions are such that sand leaves the pile only at $x=N$. The height in segment $n$ is denoted by $h(n)$, and

$$
Z(n)=h(n)-h(n+1)
$$

represents the height difference between successive positions on the sand pile. Sand is added randomly to one of the $N$ segments, every such addition to causing the changes

$$
\begin{aligned}
& Z(n) \rightarrow Z(n)+1, \\
& Z(n-1) \rightarrow Z(n-1)-1 .
\end{aligned}
$$

Addition of sand does not cause any disturbance, unless the height difference $Z(n)$ exceeds a critical value $Z_{c}$. If that happens, one unit of sand tumbles to the lower level i.e.

$$
\begin{aligned}
& Z(n) \rightarrow Z(n)-2, \\
& Z(n-1) \rightarrow Z(n-1)+1, \\
& Z(n+1) \rightarrow Z(n+1)+1
\end{aligned}
$$




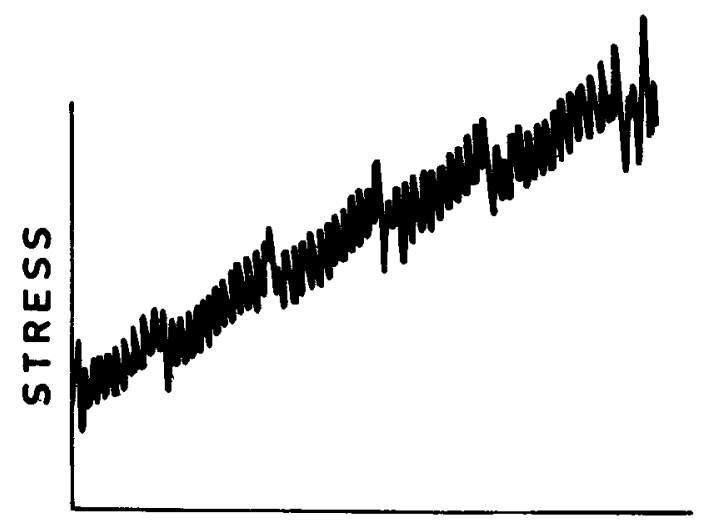

(a)

\section{TIME}

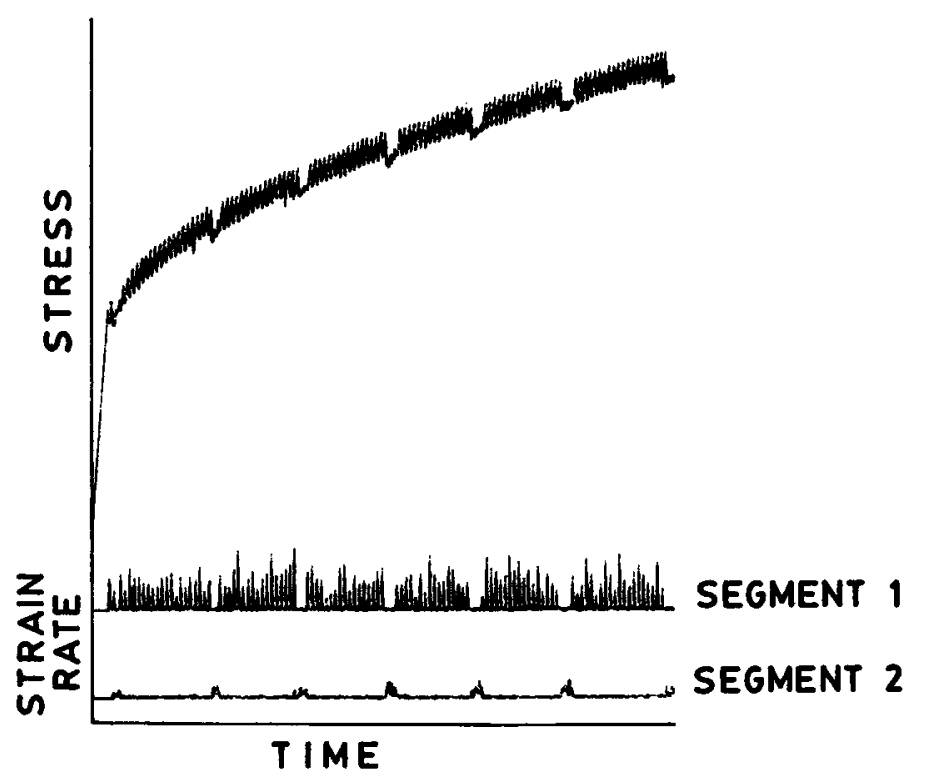

(b)

Figure 22. Bunching effects. (a) shows the results for austenitic stainless steel (Rodriguez 1984) while (b) shows the simulated curve along with the plastic strain rates.

It could happen that a disturbance so created could trigger further flow down the slope. In the simulation, fresh sand is not added until the effects of the disturbance so created have died down.

The quantities monitored are (i) the mass of the pile, and (ii) the amount of sand flowing past the open end. Results obtained with $N=30$ and $Z_{c}=7$ are shown in figure 23. Here (a) shows the mass of the sandpile as a function of time. Initially the mass continuously builds up but later there are fluctuations due to flows triggered by instabilities. The flow of grains from the pile is uneven both with respect to the quantity of flow and the time of occurrence. This stochastic feature is worthy of note as also the similarity of figure 23 (a) to the stress-strain curve in the case of 


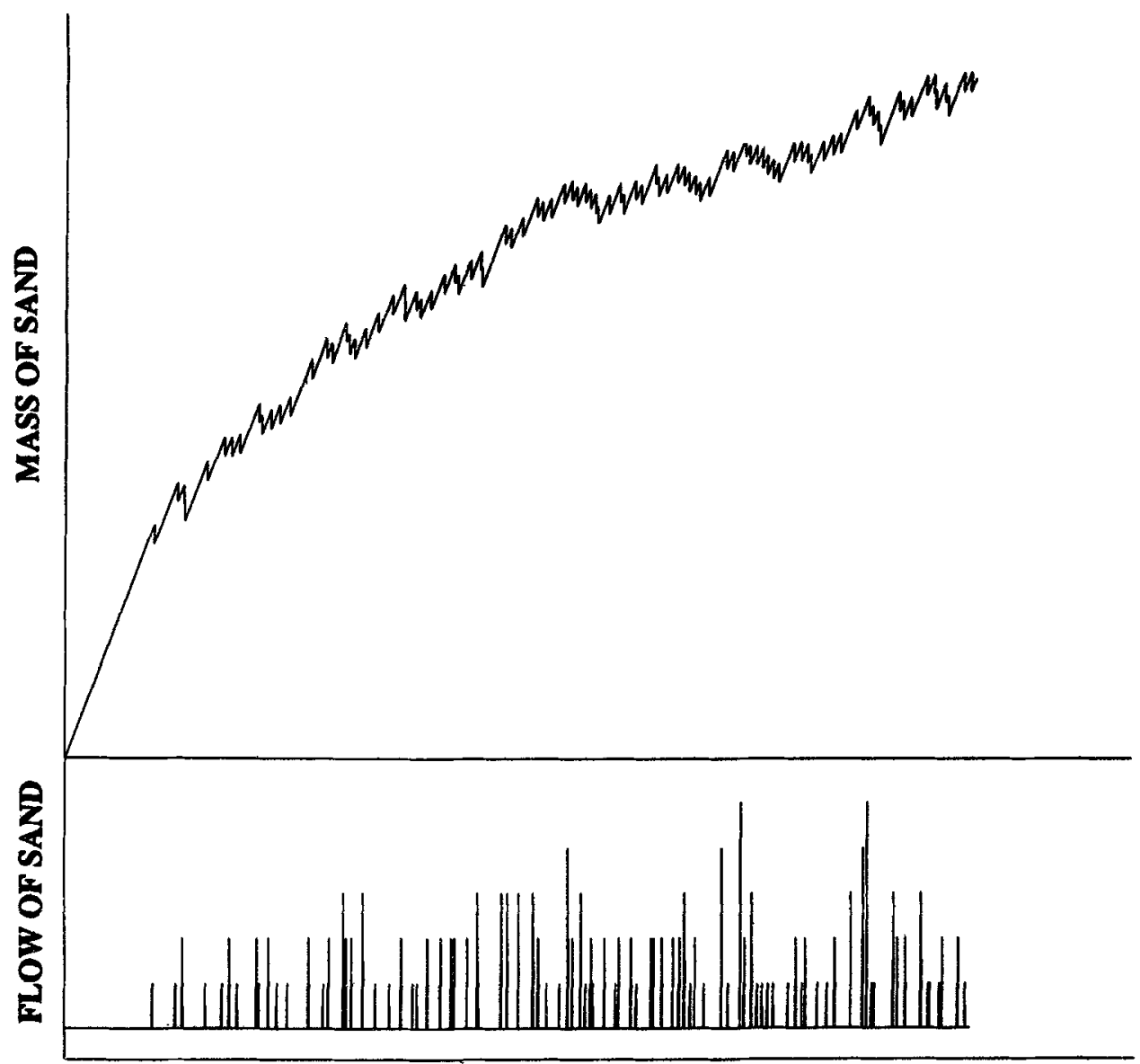

\section{STEPS}

Figure 23. This figure shows the results of our simulation studies on sand flow in a onedimensional sandpile. (a) shows the mass of the pile as a function of time while (b) depicts the outflow. Naturally, the mass of the pile drops abruptly whenever there is outflow. The rules of the game automatically ensure a built-in randomness. The purpose of this exercise is mainly to draw attention to the potentiality of the method of cellular automata to simulate dislocation flow in a strain gradient. That the method is very powerful has recently become evident from studies on fluid flow.

jerky flow. Nevertheless, one might legitimately wonder about the relevance of sand flow in a pile to the present studies. We believe that aspects of dislocation population changes and dynamics could conveniently be studied by the method of cellular automata (Wolfram 1983) used in the sand pile studies. Indeed, fluid flow has been similarly studied using massively parallel computers. While in some respects such a study might appear simplistic vis-a-vis the present problem, it is pertinent to point out that Bak et al (1988) have drawn attention to various features of universality which obtain not only in sand avalanches but equally so in plate tectonics and earthquakes. It is the latter which is worthy of note in the present context. Admittedly, as in two-dimensional sand piles, universality features would 
be seen only in idealized circumstances. Even so, methods of cellular automata are worth a serious try.

\subsection{General remarks}

In this section we explore, albeit cursorily, the simulation of inhomogeneous yielding according to various models. To our knowledge, this is the first time such results have been reported although the importance of inhomogeneity has been stressed by several authors in the past. At best, only qualitative conclusions have been drawn earlier, as paraphrased in subsection 5.1 .

To start with, some of the qualitative predictions made earlier by Penning have been verified by us by an appropriately constructed model. However, since this model does not explicitly allow for dislocation flow, an alternate model was then explored. Admittedly, our simulation studies have merely scratched the surface. Once one introduces many domains into the analysis, the degrees of freedom available increase considerably. Obviously, by manipulating these suitably one can endeavour to make the simulated yield curves come close in appearance to those obtained in experiments. We have refrained from such an arduous exercise in this case because the effort involved is not quite justified by the quality of models of inhomogeneous yielding currently available. But given a good starting model, there is no question that simulation can be carefully tuned to achieve agreement with experiment. We have also studied flow in a one-dimensional sand pile which suggests that perhaps detailed scenarios of dislocation flow could be investigated via cellular automata.

\section{Summary and conclusions}

The essential message of this paper is that relevant information about deformation mechanics can be suitably codified into a simulation algorithm capable of predicting yield curves, complete with irregularities. Obviously the algorithm is only as good as the model on which it is based. While we do not make exaggerated claims about our model, it is obvious that whatever we have used has been able to mimic many features observed in experiments. Two significant features of the present work are (i) the recognition given to stochastic effects and (ii) the attempt to actually simulate inhomogeneous yielding, albeit with a modest model.

That serrated yielding is basically an inhomogeneous process has been widely recognized. At the same time, it is frustrating that the details of this inhomogeneous processes cannot be reconstructed from an analysis of experimental data as obtained at present. This of course is to be expected. Clearly, the obvious way out is via computer simulation. As in other areas of science and engineering, computer simulation can be a valuable tool for refining detailed models and improving our understanding of inhomogeneous yielding. Some of the present gaps in understanding vis-a-vis the inhomogeneity of yielding have already been pointed out. It is our belief that large scale use of simulation could, in course of time, conceivably lead to a satisfactory model with good predictive capability. In turn this could be made use of in material technology to determine in advance the material behaviour under complex conditions of material processing (meaning complex trajectories in $\sigma-\dot{\varepsilon}-T$ space). 
In the existing literature, the focus thus far has mostly been on why and when serrations occur. Thus it is that one finds much discussion on the connection between serrations and negative strain-rate sensitivity. Without minimizing the importance of establishing such correlations, we would like to observe that by contrast scant attention has been paid to details such as (i) the shape of the yield drop pulses, (ii) the amplitude distribution of the pulses, and (iii) their temporal spacing i.e. power spectra. It is our submission that much information is buried in these hitherto neglected details also. Examples abound in other branches of science and engineering wherein such analysis has been exploited to learn details otherwise not easily accessible.

Our advocacy of simulation is not meant to imply that it is in an activity which can be carried on in isolation. As earlier indicated, simulation thrives on modelling and one hopes the power of the computer to unravel the outcome of intricately linked equations, would act as a spur to modelling. At the same time, it would also be a good idea to be abreast of related developments such as self-induced criticality. Even more important, particular attention must be paid to obtaining experimental information about spatial inhomogeneity of the yielding process, preferably on a real-time basis (Kubin et al 1986 also have made similar suggestions). Many schemes for doing this could be envisaged. For example, one could, during the tensile test, rapidly raster scan the surface of the test specimen using a laser beam and pickup inhomogeneities in the yielding via fluctuations in the reflected intensity. It might also be interesting to combine yield experiments with studies on acoustic emission. It is already known (e.g. Dunegan and Harris 1969), that acoustic emission rate exhibits a strong peak during the transition from elastic to plastic deformation, reminiscent of peaks observed during the study of critical phenomena. Acoustic emission pulses are like earthquake signals and thus it would be interesting to study the character of their pulses during serrations and look for correlations with yield drop. Such a study might be fruitful in terms of revealing more about slippages occurring across the sample, which in turn could be related to simulation studies a la Bak et al. These are but samples of what could be attempted in laboratory experiments, and obviously there are many other possibilities.

In conclusion, we observe that serrated yielding is a problem involving spatial and temporal patterns. As is well-known, such patterns are not unusual when systems have nonlinearities and are driven far from equilibrium (Haken 1983). Indeed the occurrence of such patterns has been extensively studied in widely ranging fields. There has been some appreciation of nonlinearities, instabilities, pattern formation and the like vis-a-vis deformation (e.g. Ananthakrishna and Sahoo 1981; Ananthakrishna and Valsakumar 1982; Valsakumar and Ananthakrishna 1983; Kubin et al 1984), but by and large the effects of inhomogeneity have largely been ignored in the investigations just cited. In short, the subject has not received the in-depth investigation it merits, both in terms of spatial dependence and with respect to the constitutive equations governing the material parameters entering the mechanical equation of state. In our view, the evolution of the subject has to be through an intensive interplay of modelling, simulation and laboratory experiments. The well-known series on synergetics amply demonstrates how fruitful such an exercise has been in physics, chemistry and biology. It is our hope that with the widespread availability of workstations with considerable built-in computing power and graphic capability, material scientists would not lag behind their counterparts in other disciplines. 


\section{Acknowledgements}

This work was commenced several years ago while both of us were at the Indira Gandhi Centre for Atomic Research. We are grateful to that organization for the support received. We would particularly like to thank Dr P Rodriguez and his colleagues for sharing some of their data with us and patiently explaining the various features involved. We have also benefitted much from conversations with Prof. P Rama Rao and Dr J L Strudel. One of us (GV) would like to express his gratitude to Prof. Sir Alan Cottrell, FRS and to Prof. F R N Nabarro, FRS for helpful correspondence and encouragement. Mr G Athithan kindly prepared for us the figures involving computer graphics.

\section{Appendix}

In the simulation, the coupled first-order differential equations were solved using Euler's method (Maron 1982). For convenience, the various parameters were scaled as necessary. Some of the important parameters and the ranges of values used are given in table Al. The stability of the solution process was ensured by the proper choice of the time step $\Delta t$.

Table A1.

\begin{tabular}{|c|c|c|c|}
\hline Parameter & Description & $\begin{array}{l}\text { Range of values of sca- } \\
\text { led variables used in } \\
\text { simulation }\end{array}$ & $\begin{array}{l}\text { Corresponding value/ } \\
\text { range of values in typi- } \\
\text { cal real materials }\end{array}$ \\
\hline$\rho_{i}$ & Initial dislocation density & $1-1000$ & $\begin{array}{c}10^{6}-10^{8} \\
\text { (annealed metals) }\end{array}$ \\
\hline$\rho_{f}$ & Final dislocation density & $500-100000$ & $\begin{array}{c}10^{10}-10^{11} \\
\quad \text { (iron) }\end{array}$ \\
\hline$\Omega$ & $\begin{array}{l}\text { Coefficient related to re- } \\
\text { mobilization rate }\end{array}$ & $0.01-1$ & $\begin{array}{l}0 \cdot 01-0 \cdot 06 \\
\text { (iron) }\end{array}$ \\
\hline$\alpha$ & Work hardening coefficient & $1-10$ & $\begin{array}{l}0-0.5 \mathrm{E} \\
\text { (E is the elastic } \\
\text { modulus) }\end{array}$ \\
\hline$m$ & Work hardening exponent & $0.3-0.7$ & 0.5 \\
\hline$P$ (sèe figure 3 ) & Upper critical stress & $6 \cdot 5-8 \cdot 5$ & $\begin{array}{c}4.5 \mathrm{~kg} / \mathrm{mm}^{2} \\
\text { (Vanadium-carbon) }^{*}\end{array}$ \\
\hline$Q$ (see figure 3 ) & Lower critical stress & $5 \cdot 5-7 \cdot 5$ & $\begin{array}{c}1 \mathrm{~kg} / \mathrm{mm}^{2} \\
\text { (Vanadium-carbon) }^{*}\end{array}$ \\
\hline
\end{tabular}

* Corresponds roughly to the median value of the range in scaled units.

\section{References}

Ananthakrishna G and Sahoo D 1981 J. Phys. D14 2081

Ananthakrishna G and Valsakumar M C 1982 J. Phys. D15 L 171

Ananthakrishna G and Valsakumar M C 1983 Phys. Lett. A95 69

Baird J D 1973 in Inhomogeneity of plastic deformation (Metals Park: Am. Soc. Metals)

Bak P, Tang C and Wisenfeld K 1988 Phys. Rev. A38 364

Balakrishnan V, Dattagupta S and Venkataraman G 1978 Philos. Mag. A37 65

Bergstrom Y and Roberts W 1971 Acta Metall. 19815

Beukel van den A 1975 Phys. Status Solidi A30 197

Blanc D and Strudel J L 1987 in Constitutive relations and their physical basis (Roskilde: RISO National Laboratory) 
Brindley B J and Worthington P.J 1970 Metall. Rev. 15101

Cottrell A H 1953 Dislocations and plastic flow in crystals (Oxford: Clarendon Press)

Dunegan H L and Harris D O 1969 Ultrasonics 7160

Franck U F 1974 in Physical chemistry of oscillatory phenomena (London: Faraday Society) p. 137

Freidel J 1964 Dislocations (Oxford: Pergamon)

Haken H 1983 Advanced synergetics (Berlin: Springer Verlag)

Hall E O 1970 Yield point phenomena in metals and alloys (New York: Plenum)

Keh A S, Nakada Y and Leslie W C 1968 in Dislocation dynamics (eds) A H Rosenfeld, G T Halm, A L Bement and R I Jaffee (New York: McGraw Hill) p. 381

Kocks U F 1981 Progr. Mater. Sci. Chalmers Ann. vol. 185

Kocks U F, Cook R E and Mulford R A 1985 Acta Metall. 33623

Kubin L P, Chihab K and Estrin Y 1986 in Patterns, defects and microstructures in non-equilibrium systems (ed.) D Walgraef (Martinus Nijhoff)

Kubin L P and Estrin Y 1985 Acta Metall. 33397

Kubin L P and Estrin Y 1986 J. Phys. (Paris) 47497

Kubin L P, Estrin Y and Spiesser Ph 1984 Res. Mechanica 1025

Kubin L P, Spiesser Ph and Estrin Y 1982 Acta Metall. 30385

Leslie L J and Cuddy W C 1972 Acta Metall. 201157

Lucke K and Mecking H 1973 in Inhomogeneity of plastic deformation (Metals Park: Am. Soc. Metals)

MacDonald D K C 1962 Noise and fluctuations (New York: Wiley)

Maron M J 1982 Numerical analysis: A practical approach (New York: Macmillan)

Mulford R A and Kocks U F 1979 Acta Metall. 2771125

Nabarro F R N, Basinski Z S and Holt D B 1964 Adv. Phys. 13193

Neelakantan K and Venkataraman G 1983 Acta Metall. 3177

Papoulis A 1965 Probability, random variables and stochastic processes (New York: MacGraw Hill)

Penning P 1972 Acta Metall. 201169

Pink E and Grinberg A 1981 Mat. Sci. Eng. 511

Rodriguez P 1984 Bull. Mater. Sci. 6653

Schenzle A and Brand H 1979 Phys. Rev. A20 1628

Schwarz R B and Funk L L 1985 Acta Metall. 33295

Strudel J L 1984 in Japan-France seminar on fundamental aspects of mechanical properties (Tokyo: Dept. of Material Science, University of Tokyo) 169

Valsakumar M C and Ananthakrishna G 1983 J. Phys. D16 1055

Venkataraman G 1979 Trans. Indian Inst. Met. 32435

Venkataraman G 1982 in Mechanical and thermal hehaviour of materials (Amsterdam: North Holland) p. 278

Wolfram S 1983 Rev. Mod. Phys. 55601

Yoshinaga A, Toma K, Abe K and Morozumi S 1971 Philos. Mag. 231387

Ziel van der A 1970 Noise-Sources, characterization, measurement (New Jersey: Prentice Hall)

Note added in proof

After submitting our paper for publication we became aware of the following papers whose contents are pertinent to the present work. We are grateful to the authors for drawing our attention to them:

Aifantis E C 1983 in Mechanics of dislocations (Metals Park: Am. Soc. Metals)

Aifantis E C 1984 J. Eng. Mat. Tech. 106326

Aifantis E C 1985 in Dislocation in solids (Tokyo: Univ. of Tokyo Press)

Aifantis E C 1987 Int. J. Plasticity 3211

Aifantis E C 1989 in Proc. International Conference on Constitutive Laws for Engineering Materials (ed.)

F Jinghong and $S$ Murakami (Oxford: Pergamon)

Estrin Y 1988 in Nonlinear phenomena in materials science (Aedermannsdorf: Trans Tech.)

Estrin Y and Kubin L P 1989 J. Mech. Beh. Mater. 2255

Estrin Y and Kubin L P 1991 Mater. Sci. Eng. A137 125

Zbib H M and Aifantis E C 1988 Scr. Met. 22703

Zbib H M and Aifantis E C 1988 Scr. Met. 22 1331 